\title{
RICORDO DEL m.e. GIAMPIERO LANDENNA
}

\author{
s.c. GIORGIO DALL'AGLIO (*)
}

(Adunanza del 5 ottobre 2017)

Saluto il presidente, i soci, i familiari e gli amici di Giampiero Landenna, e ringrazio per l'opportunità che mi viene data di ricordare un caro amico.

Comincio coi miei ricordi, che risalgono a più di sessanta anni fa. Nel 1954 ero alla Scuola Normale Superiore di Pisa e lavoravo alla mia tesi di laurea, sotto la guida del professor Alessandro Faedo; ma l'argomento della tesi era proposto dal professor Giuseppe Pompilj della Facoltà di Statistica (più precisamente di Scienze Statistiche Demografiche e Attuariali di Roma, poi La Sapienza). Giampiero Landenna mi venne a trovare; preparava la tesi di laurea con il professor Pompilj, di cui poi anche io divenni allievo. E' a Pompilj che dobbiamo la prima conoscenza e la lunga e proficua amicizia che per tanti anni ci ha legato. Noi avevamo per lui affetto e devozione, egli purtroppo morì troppo presto.

Tornando alla nostra vita di studenti. siccome gli argomenti delle due tesi erano collegati dovevamo confrontarne gli sviluppi. Cominciò così una frequentazione che, andando al di là della motivazione scientifica, continuò senza interruzioni. Ci siamo visti a Roma, dove lui veniva per proseguire le sue ricerche con Pompilj, che Landenna ha sempre considerato il suo maestro. Lo rivedevo in queste occasioni, e avevamo anche la possibilità di studiare insieme, esaminando diversi problemi relativi alle ricerche che conducevamo. Questi incontri sui nostri lavori 
e sugli aspetti che li legavano sono continuati per diversi anni. Più tardi ci incontravamo anche a Milano, dove mia moglie Laura e io siamo stati più volte ospiti di Giampiero e sua moglie Elena. E la mia riconoscenza va a lui per la mia appartenenza a questo Istituto. L'ho rivisto anche poco tempo prima della sua scomparsa.

E sento qui il dovere di dare qualche cenno sulle importanti realizzazioni del nostro comune maestro, che non sono molto conosciute.

Giuseppe Pompilj aveva cominciato la sua attività di ricerca in geometria, sotto la guida del professor Federico Enriques. Ma la sua vita scientifica ebbe una svolta nel campo di prigionia inglese in India in cui fu detenuto durante a guerra. Perché la sua attenzione fu attratta da alcuni scritti trovati nella piccola biblioteca del campo. Erano sul notevole sviluppo della statistica nel mondo anglosassone, poco conosciuto in Italia a causa della guerra, ma anche per la tendenza autarchica dovuta al fascismo. Al ritorno in Italia fu ternato in un concorso per la cattedra di geometria; ma mosso dai suoi nuovi interessi si rivolse a Corrado Gini, insigne cultore di statistica, oltre che di demografia e sociologia, che riconobbe l'importanza dei nuovi argomenti. Cosicché Pompilj nel 1948 fu chiamato alla Facoltà di Statistica, alla cattedra di Geometria per la quale era qualificato, ma con l'incarico di Calcolo delle Probabilità per insegnare questa materia e anche Statistica Matematica.

Pompilj si dedicò intensamente all'insegnamento e alla promozione. Invitò docenti e studiosi stranieri, e inviò all'estero con borse di studio i suoi allievi. Istituì presso la Facoltà l'Istituto di Calcolo delle Probabilità, ed anche la Scuola di Perfezionamento in Ricerca Operativa. Morì a 54 anni nel 1968 per un aneurisma. In venti anni aveva contribuito alla diffusione in Italia della Probabilità, promosso la statistica matematica e l'inferenza statistica, "importato" in Italia la Ricerca Operativa, allora praticamente conosciuta solo nelle forze armate, per influsso degli stati alleati. Soprattutto aveva creato un nutrito gruppo di allievi di alto livello, molti dei quali dopo la sua prematura scomparsa ottennero una cattedra, parecchi in Statistica, dove lui non aveva mai avuto autorità accademica.

Giampiero Landenna nacque a Milano il 26 Dicembre 1923 da Arturo Valentino e Maria Contessi, persone umili e poco abbienti, ma dotate di grande intelligenza e di amore per Giampiero e per la sorellina nata dieci anni dopo, ai quali fornirono una profonda educazione cul- 
turale e morale. A tredici anni rimase orfano della madre, e diventò un sostegno ed un punto di riferimento per il padre e la sorella.

E' necessario sottolineare la notevole forza d'animo e la grande capacità lavorativa di Landenna, che lo hanno sostenuto in questo periodo e gli hanno permesso di percorrere la sua brillante carriera. Accanto ad esse vanno ricordate la profonda integrità e l'onestà intellettuale, il suo non voler mai scendere a compromessi (cose che gli sono valse non poche inimicizie), "una rigorosa serietà professionale e una grande determinazione" (Alberto Martinelli nel volume a lui dedicato, n. A1 della bibliografia). Si esprimeva in più campi: la famiglia, il lavoro,la musica; a tutto si interessava con intelligenza e entusiasmo.

Nel 1942 conseguì il diploma di Ragioneria, che gli permise di lavorare per aiutare la famiglia, ma anche di proseguire tenacemente e con fatica il suo percorso di studio. Sapeva dove voleva arrivare, e frequentando la scuola serale ottenne il diploma di maturità scientifica che gli permise di accedere all'università. Con la guerra fu richiamato, in artiglieria; durante il periodo di occupazione tedesca riuscì a sottrarsi al reclutamento per la Repubblica di Salò, rimanendo nascosto nelle campagne veronesi dove si guadagnava da vivere dando lezioni di matematica. Rientrò a Milano rifugiandosi presso il convento di Santa Maria delle Grazie, aiutato da padre Alfonso Glanzer che era stato direttore spirituale di lui e Elena. Trovò il modo di sdebitarsi per l'ospitalità ricevuta, perché salvò la pala della Madonna delle Grazie della cappella sinistra, portandola in bicicletta presso un convento di suore a Monza.

Intanto nasceva la sua famiglia: sposato nel 1950 con Elena Neri Mana, nacquero Marialucia, chiamata familiarmente Nanni, nel 1951, Paolo nel 1954 e Isabella nel 1958. Il matrimonio era basato su una fede profonda: insieme andavano a messa la domenica, insieme recitavano il rosario la sera. Elena era per lui la madre che aveva perso troppo presto, la confidente, soprattutto la moglie amata e un prezioso aiuto. E Giampiero, assieme a Elena, trasmise ai figli l'educazione che aveva ricevuto a suo tempo; senza le restrizioni economiche di allora, poterono assicurare loro ottime scuole, assieme allo studio delle lingue in varie istituzioni. $\mathrm{Li}$ accompagnarono poi fino alla laurea: Marialucia in matematica, Paolo in medicina e odontoiatria, Isabella in Lingue e Letteratura Tedesca.

Naturalmente Landenna continuava i suoi studi. La figlia Marialucia ricorda il padre che per preparare la tesi partiva alle tre di notte da Milano Marittima per raggiungere il professor Pompilj, anche 
lui in vacanza. Dopo anni di vita operosa e felice, la salute degenerò. Lo colse un'insufficienza renale gravissima, e poi una malattia degenerativa alla mandibola che tra l'altro gli rendeva difficile parlare. Gli restavano accanto i figli. Soprattutto gli fu di grande aiuto la figlia Nanni, che abitava nello stesso palazzo, al piano sopra al suo. Ricordo che negli ultimi tempi solo con il suo aiuto era possibile parlare con lui. Giampiero era persona comunicativa, piena di interessi; e certo per lui era una grande sofferenza non poter parlare. Negli ultimi mesi la figlia Nanni passava tutte le notti presso di lui, ascoltando a fatica i suoi ricordi. Morì il 20 Dicembre 2016, lo stesso giorno di suo padre.

Ma si era realizzata la benedizione del salmo 128 "possa tu vedere i figli dei tuoi figli”, con i suoi tre figli e cinque nipoti; era anche diventato bisnonno, e tre giorni prima di morire poteva sostare accanto alla piccola Vittoria, con la mano sulla sua culla.

Dopo l'impegno faticoso degli inizi, la sua attività scientifica proseguì regolarmente. Ci fu l'incontro con il professor Marcello Boldrini, che Landenna ha amato di un amore filiale, e che ha guidato la sua vita accademica. Una grande amicizia è nata anche tra Elena e la signora Boldrini, donna raffinata, colta e valente pittrice, che ha anche regalato alcuni suoi acquerelli e una punta secca, che hanno arricchito il suo studio.

Landenna è stato molto legato anche al professor Albino Uggé, non solo per motivi accademici; frequentava la casa come un figlio, e il professore lo nominò suo esecutore testamentario. Tra gli amici ricordo anche Amato Herzel, Mario Di Bacco e Alighiero Naddeo. Con tutti loro ha svolto ricerche in collaborazione e ha anche scritto opere o articoli.

Anche il suo percorso accademico fu regolare. Conseguì a Milano il Diploma di Statistica, e poi nel 1955 la laurea in Scienze Statistiche e Attuariali presso la Facoltà di Statistica di Roma, l'unica che la rilasciava all'epoca. Spostò poi la sua attività all'università di Milano. Cominciò nell'anno accademico 1961/62 come assistente volontario di statistica nella Facoltà di Scienze Economiche dell'università Cattolica; nel 1963 conseguì la libera docenza. Dall'anno accademico 1963/64 al 1974/75 tenne senza interruzione corsi di Statistica, Statistica Sociale, Statistica Giudiziaria, a volte più di uno nello stesso anno, ricoprendo anche dal 1971 gli incarichi di Direttore della Scuola di Statistica e Direttore dell'Istituto di Statistica.

Contemporaneamente insegnava a Genova, dove nel 1966 ottenne l'incarico del corso di Statistica e la direzione dell'Istituto di 
Statistica. Intanto entrò tra i vincitori del concorso per la cattedra di Statistica. Nel 1970 vinse il concorso per assistente di Statistica presso la Facoltà di Economia e Commercio dell'Università Cattolica del Sacro Cuore, ma nello stesso anno fu chiamato alla cattedra di Statistica nella Facoltà di Economia e Commercio dell'Università Ca' Foscari di Venezia. Dopo un anno si trasferì a Genova, continuando così con la cattedra l'insegnamento già tenuto come incaricato. Conservava intanto il suo incarico presso l'Università Cattolica, con la direzione dell'Istituto di Statistica e della Scuola di Statistica, succedendo al professor Uggè dopo la sua scomparsa.

Nel 1974 si spostò a Milano, la sua sede definitiva, presso la Facoltà di Scienze Politiche dell'Università di Milano. All'insegnamento univa una infaticabile attività di direzione e organizzazione. Promosse l'istituzione della Scuola di Statistica, che venne poi trasformata in Corso triennale di Statistica per assumere la forma definitiva di Corso di laurea in Scienze Statistiche, Demografiche e Sociali nell'Università di Milano Bicocca, di cui fu nominato direttore. In seguito, ancora per sua iniziativa, nacque nell'Università di Milano la Facoltà di Scienze Politiche.

E qui naturalmente continuò la sua attività di ricerca e di insegnamento ad alto livello. Ebbe numerosi allievi; la più vicina a lui fu Donata Marasini, che nel volume in suo onore (n A1), esprimendo la sua commossa riconoscenza, ricorda tra gli altri allievi Giancarlo Blangiardo, Pieralda Ferrari, Marco Martini, Giorgio Vittadini, oltre ad altri più giovani. Avviò tutti alla ricerca, e assieme a molti di loro scrisse articoli scientifici e anche libri.

Il suo servizio all'università ebbe fine nel 1998.

Bisogna ricordare anche l'attività esterna all'università, per esempio con l'insegnamento presso la Scuola Enrico Mattei di Studi Superiori sugli Idrocarburi. Fu attuario di importanti istituti bancari; nella Cariplo fu anche presidente del Collegio degli Attuari.

Un prestigioso incarico è stato la consulenza per la Divisione Statistica della Comunità Europea.

$\mathrm{Fu}$ membro di diverse istituzioni scientifiche, oltre a questo Istituto, come la Società di Statistica, in cui fece parte anche del Consiglio di Amministrazione, la Bernoulli Society, e il prestigioso Istituto Internazionale di Statistica. Nel1986 gli venne conferita la Medaglia d'oro ai Benemeriti della Scuola, della Cultura e dell'Arte. Nel 2000 fu nominato Professore Emerito. 
Nella vita di Giampiero Landenna ha avuto un posto di rilievo la musica. Già all'età di sette anni, malgrado le ristrettezze economiche, la mamma gli comprò un violino e lo iscrisse ad una scuola di musica; e a lui e alla sorella i genitori instillarono l'amore per l'arte. Col tempo diventò un dilettante di buon livello; teneva serate musicali con amici anche loro di buon livello, notevoli anche per la dimensione umana e culturale: uno di loro parlò alla famiglia degli gli orrori dei lager e delle persecuzioni degli ebrei, e fece conoscere la cultura ebraica. Suonò anche più volte con un primo violino della Scala, ed ebbe la straordinaria occasione di suonare uno Stradivari presso la famiglia Costa, quando insegnava a Genova. Ha anche lasciato una piccola collezione di strumenti: due violini e una viola

La ricca produzione scientifica di Giampiero Landenna ( 75 voci) è molto articolata; tre di essi sono apparsi sugli atti di questo Istituto. Vediamo anzitutto i due filoni ai quali lo aveva avviato Giuseppe Pompilj: lo studio delle relazioni tra distribuzioni statistiche, che Pompilj riprendeva dalla scuola statistica di Corrado Gini, e la statistica matematica, facente capo alla scuola statistica anglosassone.

Il primo settore, poco numeroso (7 lavori), comincia con l'articolo sulla dissomiglianza, derivante dalla tesi di laurea, e prosegue in diverse direzioni, in particolare la connessione e la cograduazione.

Il secondo settore, con 28 lavori (qualcuno in collaborazione), costituisce la parte più cospicua e rilevante della sua produzione, esplorando diversi ambiti dell'inferenza statistica. Alcuni lavori riguardano l'impostazione e qualche aspetto generale, come il piano degli esperimenti e la verifica delle ipotesi. Segue lo studio di vari modelli (Pascal, Bernoulli, Poisson).

Allo stesso gruppo appartengono l'analisi della varianza, con la stima della varianza e gli errori di prima specie, e inoltre l'analisi sequenziale, i test non parametrici e l'impiego del chi quadro. Vengono esaminati anche alcuni aspetto della teoria dei campioni.

Diversi di questi lavori contengono applicazioni alle ricerche di mercato.

Allo studio delle distribuzioni di statistica e probabilità sono dedicati 6 lavori, che trattano in particolare la distribuzione di Polya, l'ipergeometrica, e i miscugli di variabili casuali.

Un gruppo nutrito (10 lavori, diversi in collaborazione) si occupa di applicazioni a problemi socio-economici. Anzitutto scambi commer- 
ciali con l'estero, dell'Italia, ma soprattutto della Comunità Europea, con paesi o gruppi di diverse parti del mondo, dall'Africa ai paesi neolatini e altri. Non manca l'esame di problemi demografici.

Restano 11 scritti di varia natura. Oltre a ricordi di colleghi e di alcune personalità, sono trattati alcuni argomenti generali sulla statistica.

Alla diffusione delle ampie conoscenze da lui maturate nella sua lunga carriera si rivolgono ben 13 libri. Uno è rivolto specificamente al suo insegnamento; gli altri sono stati pubblicati tutti da editori prestigiosi: Franco Angeli (n. 16 della bibliografia, con applicazioni alla produzione industriale); Giuffré (probabilità e inferenza, n. 22 e 23; statistica matematica, n. 34 e 35; le diverse concezioni della probabilità, 59); il Mulino (statistica descrittiva n. 56, probabilità n. 68, inferenza statistica 63, 69, 70); Cacucci (metodi di stima, n.64).

Concludo ringraziando ancora l'Istituto che mi ha permesso di ripercorrere la lunga stagione dell'amicizia tra me e Giampiero, e ricordare quanto ho ricevuto di ricchezza umana e culturale. Sono grato a Nanni per il suo inestimabile aiuto in questo mio compito. E grazie a voi tutti che mi avete ascoltato.

\section{ELENCO DELLE PUBBLICAZIONI}

1. "La dissomiglianza", Statistica, 1, 1956.

2. "Teoria degli esperimenti ed analisi di mercato", Studi di mercato, 2, 1957.

3. "Osservazioni sulla connessione", Statistica, 4, 1957.

4. "Lo schema probabilistico a due stadi semplici", Statistica, 4, 1959.

5. "Analisi delle frequenze nelle ricerche di mercato", Studi di mercato, 1, 1960.

6. "I tests 'non parametrici' basati sulle frequenze", Istituto di Calcolo delle Probabilità, Roma, 1960.

7. "Analisi degli ordinamenti nelle ricerche di mercato", Studi di mercato, 1, 1961.

8. "L'indice semplice di dissomiglianza tra campioni ottenuti con estrazione in blocco", Statistica, 3, 1961.

9. "Impostazione Bayesiana dell'analisi sequenziale", Statistica, 4, 1961.

10. "Le plan d'experience”, Centre National de la Recherche Scientifique, Paris, 1961.

11. "Considerazioni sulla funzione di potenza del test Chi quadrato", Giornale degli Economisti, 3, 1963.

12. "Un problema di impiego del test Chi quadrato, in relazione alla sua funzione potenza”, Atti del V Convegno A.I.C.Q., 1963.

13. "L'analisi sequenziale negli universi rettangolari", Statistica, 1, 1964.

14. "Lo schema probabilistico di Pòlya-Lexis-Laplace", Giornale degli Economisti, 1, 1966. 
15. "Gli schemi probabilistici di Pòlya-Poisson e Pòlya-Coolidge", Atti dell'Istituto Lombardo, 1966.

16. "Metodi Statistici nella ricerca scientifica e nella programmazione industriale", Milano, Franco Angeli, vol. I, 1966.

17. "La variabile casuale di Pòlya come miscuglio di Multinomiali", Atti dell'Accademia dei Lincei, 1966.

18. "Gli schemi di Bernoulli e di Poisson con numero di prove variabile aleatoriamente”, Atti dell'Istituto Lombardo, 1967.

19. "Su alcune variabili casuali miscuglio", Atti dell'Accademia Ligure, 1967.

20. "Un metodo per il controllo dell'errore di prima specie nell'analisi della varianza", Giornale degli Economisti, 2, 1967.

21. "Stima della varianza nel campionamento a due stadi", Atti dell'Istituto Lombardo, 1967.

22. "Teoremi sui parametri per la verifica delle ipotesi", Giuffrè, Milano, 1968.

23. "Teoria delle variabili casuali”, Milano, Giuffrè, 1968.

24. "La legge di Pascal ed i ritardi nel gioco del lotto", Giornale degli Economisti, 1969.

25. "Lezioni di Statistica”, parte III e IV, C.E.L.U.C., Milano, 1969.

26. "Un nuovo criterio per condurre l'analisi della varianza nel caso di fattori ad effetti variabili”, Giornale degli Economisti, 3, 1969.

27. "Un indice sintetico della Congiuntura Italiana per il Commercio con l'estero, nel periodo 1960-1968”, da la “Componente estera nell'Economia Italiana”, vol. II, 1969.

28. "Lezioni di Statistica”, parte I e II, C.E.L.U.C., Milano, 1970.

29. "Sul minimo della varianza di una stima", Giornale degli Economisti, 1, 1972.

30. "Aspetti demografici dell'area meridionale lombarda", Pavia Economica, 2, 1972.

31. "Sui momenti della variabile casuale ipergeometrica", Atti dell'Accademia Ligure, 1973.

32. "Ricordo di Albino Uggè", Vita e Pensiero, 1973.

33. Revisione dell'opera di Marcello Boldrini "Statistica: teoria e metodi" in occasione dell'ultima ristampa.

34. "Statistica Matematica", quaderno I, Milano, Giuffrè, 1974.

35. "Statistica Matematica", quaderno 2, Milano, Giuffrè, 1974.

36. "La Statistica e lo Statistico" (in collaborazione con A. Naddeo), in Atti del Convegno "Induzione, Probabilità e Statistica", Venezia, 1978.

37. "Avondo Bodino necrologio" Annuario della Facoltà di Scienze Politiche, Milano, 1981-1982.

38. "Lo schema di campionamento secondo Pòlya e la variabile casuale di Dirichlet", Statistica, 4, 1979.

39. "Analisi della varianza e fattori ad effetti variabili", in Atti dell'Accademia Ligure, pp. 201-216, 1979.

40. "Campionamento con risposte casualizzate: un miglioramento della tecnica di S. L. Warner", Studi in onore di Libero Lenti, 1979.

41. "Un test che consente il controllo dell'errore di prima specie" (in collaborazione con Donata Marasini), in Atti della Summer International School, 1980. 
42. "Analysis of trade between the European Community and the Arab League Countries", (in collaborazione con M. Martini, G.C. Blangiardo), Eurostat, 1980.

43. "Analysis of trade between the European Community and the Latin American Countries 1965-1980", (in collaborazione con M. Martini , G. C. Blangiardo), Eurostat, 1981.

44. "A two-dimensional T-distribution and a new test with flexible type I error control", in Statistical Distribution in Scientific Work, Series C: Mathematical and Phisical Sciences, Nato Advanced Studies Institutes Series, (in collaborazione con Donata Marasini) Vol. 5, pp. 193-202, 1981.

45. "Relazione sui lavori della Commissione di Studio sul tema: "Le recenti tendenze della metodologia statistica nell'impostazione dell'inferenza”, pp. 179-187 - Atti del Convegno della Società Italiana di Statistica, vol. II - Pavia - Salice Terme 2729 aprile 1981.

46. "Schemi di campionamento a due stadi con risposte casualizzate" (in collaborazione con D. Marasini e P.Ferrari), Atti della XXI Riunione Scientifica della Società Italiana di Statistica, vol. III, Torino, 5-7 Aprile 1982.

47. "Critical Analysis of Ian Hacking's book "Logic of Statistical Inference"” (in collaborazione con A. Herzel, A. Naddeo, P. Ferrari, D. Marasini), Società Italiana di Statistica, 1982.

48. "Su uno schema di campionamento con risposte casualizzate", Rivista Internazionale di Scienze Economiche e Commerciali, vol. 29, 1982.

49. "Il campionamento con risposte casualizzate: aspetti generali", Rivista di Statistica Applicata, vol. 16, 1983.

50. "Breve nota sugli scambi intercorsi nel periodo 1970-1981 tra la comunità Europea ed i paesi aderenti alla Convenzione di Lomè”, Banca Toscana, Studi ed Informazioni, n. 3, 1983.

51. "EC: ACP Trade; A Statistical Analysis", (in collaborazione con M. Martini), 1983.

52. "Sulla costruzione dei test per la verifica di ipotesi semplici", (in collaborazione con P. Ferrari) in Annali della Facoltà di Scienze Politiche dell'Università di Milano, III - pp. 283.294, 1983.

53. "Linterscambio commerciale CEE -ACP- Finafrica" (in collaborazione con M. Martini), 1983.

54. "Un'interpretazione della "Stringency" di I. Hacking", (in collaborazione con P. Ferrari), Atti della XXXII Riunione Scientifica della S.I.S., Sorrento, 1984.

55. "Tullio Biagiotti” Rendiconti dell'Istituto Lombardo, 1988.

56. "Fondamenti di Statistica descrittiva”, Bologna, Il Mulino, 1984.

57. "Profilo scientifico e umano di Tullio Biagiotti", in Rivista Internazionale di Scienze Economiche e Commerciali, vol. 33, n. 1, pp. 1-5, Padova, Cedam, 1986.

58. "Breve excursus su alcuni test non parametrici", in Quaderni dell'Istituto di Statistica, n.38, Università degli Studi di Bari, Facoltà di Economia e Commercio, Bari, 1986.

59. "Uno sguardo alle principali concezioni probabilistiche", (con D. Marasini), Milano, Giuffrè, 1986.

60. "The theory of Connection in the Italian Conception", (in collaborazione con D. 
Marasini e E. Drago), "Italian contributions to the methodology of Statistic", Società Italiana di Statistica pp. 111-122, Padova, Cleup, 1987.

61. "Una legge di distribuzione approssimata della variabile casuale $G$ descritta dal coefficiente di cograduazione di C. Gini", (in collaborazione con A. Scagni), Metron, Rivista Internazionale di Statistica vol. XLVI, n. 1-4, pp. 71-79, 1988.

62. "An approximated Distribution of the Gini's Rank Association Coefficient", (in collaborazione con A. Scagni) Comm. Statist.-Theory meth., vol. 18, n. 6, pp. $2017-$ 2026, 1989.

63. "Metodi Statistici non parametrici", (con D. Marasini), Bologna, Il Mulino, 1990.

64. "La teoria della stima puntuale" (con Donata Marasini), Bari, Cacucci Editore, 1992.

65. "Alcune riflessioni sul tema dello "sviluppo sostenibile", Convegno su "Science, Economics and Technology for the Environment", Stresa, settembre 1994.

66. "Remarks on the issue of sustainable development", Scienza, Economia e Tecnologia per l'ambiente, Fondazione Cariplo per la Ricerca Scientifica, pp. 318326, 1995.

67. "Gerolamo Cardano e gli inizi del Calcolo delle Probabilità", Milano, CUESP, 1997.

68. "Probabilità e variabili casuali”, (con D. Marasini, P. Ferrari), Bologna, Il Mulino, - vol. I - 1997.

69. "Teoria della stima”, (con D. Marasini, P. Ferrari), Bologna, Il Mulino, voI. II, 1997.

70. "La verifica di ipotesi statistiche", (con D. Marasini, P. Ferrari), Bologna, Il Mulino, vol. III, 1998.

71. "Verso il superamento della teoria dell'equilibrio economico generale", "Disequilibrio ed equilibrio economico generale" - incontro di studio - n. 14 - pp. 5-7, Istituto Lombardo - Accademia di Scienze e Lettere, Milano, 1998.

72. "Su alcuni nuovi contributi alla teoria dei test secondo Neyman-Pearson", Statistica, 1999.

73. "Un uomo di scienza: il "Non Celebrato" matematico barnabita Don Paolo Frisi", Milano, Cuesp, 2000.

74. "Movimenti demografici e movimenti migratori: prospettive e problematiche", Il Risparmio, n. 1, 2000 (in collaborazione con G.C. Blangiardo).

75. "Su un indice di omogeneità per variabili casuali" ( con D. Marasini), Studi in onore di Angelo Zanella, Vita e Pensiero, 2002.

A1 Studi in onore di Giampiero Landenna. Giuffré 1996.

A2 Contributi alla Statistica Quaderno del Dipartimento di Statistica, Università di Milano-Bicocca, 2010. 